\title{
Über Nitroclupein.
}

Von

A. Kossel und E. L. Kennaway.

(Aus dem physiologischen Institut in Heidelberg.)

Die chemischen Eigenschaften der Proteinstoffe und ihre Beteiligung an den physiologisch-chemischen Prozessen sind nicht nur von dem Vorhandensein und der Menge der einzelnen Eiweißbausteine abhängig, sondern auch von der Anordnung derselben im Molekül. Durch diese Anordnung wird die Ausstattung des ganzen Proteinmoleküls mit freien Amido-, Carboxyl- oder Hydroxylgruppen bedingt. Wir können uns z. B. vorstellen, daß zwei Proteine, etwa Edestin und Histon, die gleiche Menge Arginin enthalten, daß aber in ersterem wenige oder gar keine Amidogruppen des Arginins in freiem Zustand vorhanden sind, während dies bei letzterem in ausgiebigem Maße der Fall ist. Die Reaktionen der beiden Proteine würden in diesem Falle sehr verschiedene sein, und das ist bekanntlich in der Tat der Fall.

Die folgenden Untersuchungen sind dazu bestimmt, Erfahrungen über die freien reaktionsfähigen Gruppen einiger Proteinstoffe zu sammeln. Im Anschluß an frühere Arbeiten über das Säurebindungsvermögen der Protamine und über die Einführung von Säureradikalen in diese einfachsten Proteine ${ }^{1}$ ) versuchten wir festzustellen, welcher Teil des Proteinmoleküls für die Einführung der Nitrogruppe zugänglich ist, und wir wandten uns auch hier zunächst zu den einfachsten Proteinstoffen, um unsere Untersuchungen später auf andere Proteinstoffe zu übertragen.

Es ergab sich hierbei, daß das Clupein in eine Nitroverbindung übergeführt werden kann, welche bei der Hydrolyse

1) cf. Hirayama, Diese Zeitschrift, Bd. 59, S. 285. 
das bisher noch nicht beschriebene Nitroarginin liefert. Wir konnten dasselbe Nitroarginin auch durch Nitrierung von Arginin gewinnen und halten es für sehr wahrscheinlich, daß das letztere ein Derivat des von Thiele beschriebenen ${ }^{1}$ ) unsymmetrischen Nitroguanidins ist, daß ihm mithin die folgende Konstitution zukommt:

$$
\mathrm{NO}_{2}-\mathrm{NH}-\mathrm{CNH}-\mathrm{NH}-\mathrm{CH}_{2}-\mathrm{CH}_{2}-\mathrm{CH}_{2}-\mathrm{CHNH}_{2}-\mathrm{COOH} \text {. }
$$

Nach dieser Auffassung würde die Nitrogruppe sowohl bei der Nitrierung des Arginins wie bei der des Clupeins in die Guanidingruppe eintreten, in gleicher Weise, wie dies bei der Nitrierung des freien Guanidins der Fall ist. Hieraus würde weiterhin zu schließen sein, daß auch im Clupein freie reaktionsfähige Guanidingruppen vorhanden sind. Dies ist wiederum in guter Übereinstimmung mit dem bisher ermittelten Säurebindungsvermögen des Clupeins, welches auch mit Wahrscheinlichkeit darauf hinweist, daß die Guanidingruppen frei und an der Peptidbindung nicht beteiligt sind. Es hat sich nämlich bei der Untersuchung der Salze des Clupeins und bei der Bestimmung seiner Alkalescenz herausgestellt, daß jeder Guahidingruppe des im Clupein enthaltenen Arginins annähernd ein basisches Äquivalent des Clupeins entspricht. Die Guanidingruppen des Clupeins verhalten sich also bezüglich der Säurebindung und bei der. Nitrierung ebenso wie die Guanidingruppe des Arginins. ${ }^{2}$ )

$\because \quad$ Die nähere Begründung dieser Auffassung, sowie die Ausdehnung dieser Untersuchung auf andere Proteine sind Gegenstand weiterer Untersuchungen im hiesigen Institut.

Darstellung und Eigenschaften des Nitroclupeins.

$\because \quad 2 \mathrm{~g}$ Clupeinsulfat werden mit $4 \mathrm{ccm}$ konzentrierter Schwefelsäure und $2 \mathrm{ccm}$ rauchender Schwefelsäure $\left(10 \% \mathrm{SO}_{3}\right)$ unter Abkühlung mit Eis zur gleichmäßig dicklichen Masse verrührt. Man fügt nun $1 \mathrm{ccm}$ rauchende Salpetersäure, welche ebenfalls abgekühlt ist, hinzu und mischt unter stetigem Abkühlen dưrch. Nảch 5-10 Minuten läßt man die dickliche Masse in

1) Liebigs Annalen der Chemie, Bd. 270, S. 1.

2) cf. Goto, Diese Zeitschrift, Bd. 37, S. 112. 
etwa $200 \mathrm{ccm}$ eisgekühltes Wasser tropfen; das Nitrocluein scheidet sich als rein weißer Niederschlag aus. Während diser Operationen darf sich weder Erwärmung noch Gasentwicking einstellen, auch soll das Präparat keine gelbe oder brane Farbe haben. Der Niederschlag ist nach einigem Stehen verreiblich, er wird in fein zerriebenem Zustand mit Wasser cusgewaschen und durch Auflösung in 2\% iger Natronlauge and Ausfällung mit Schwefelsäure gereinigt.

Das so dargestellte Produkt löst sich leicht in verdünter Natronlauge, auch geht es beim Erwärmen mit verdünter Ammoniakflüssigkeit oder mit einer Lösung von kohlensauem Natron in Lösung, die letztgenannten Lösungen trüben sich beim Erkalten unter Ausscheidung von Tröpfchen. Durch Minecalsäuren wird das Nitroclupein aus den alkalischen Lösungen wieder ausgefällt. Die den Protaminen eigene Biuretreaktion tritt auch bei diesem Körper ein.

\section{Hydrolyse des Nitroclupeins.}

$10 \mathrm{~g}$ Nitroclupein wurden mit einer Mischung von $30 \mathrm{~g}$ konzentrierter Schwefelsäure und $60 \mathrm{ccm}$ Wasser 6 Stunden arn Rückflußkühler erhitzt und nach passender Verdünnung mit Wasser mit dem Silbersulfat-Barytverfahren ${ }^{1}$ ) ausgefällt. Der Silberniederschlag wurde in verdünnter Schwefelsäure suspendiert und mit Schwefelwasserstoff zerlegt. Nachdem die Schwefélsäure durch die erforderliche Menge Baryt unter Vermeidung eines Überschusses entfernt war, wurde die Flüssigkeit eingedampft. Aus dem Rückstand schieden sich Krystalle aus, welche nach mehrmaligem Umkrystallisieren unter Zusatz von Tierkohle einen Schmelzpunkt von $227-228^{\circ}$ zeigten und deren Menge ungefähr $3 \mathrm{~g}$ betrug. Sie drehten die Ebene des polarisierten Lichtes nach rechts. Die Analyse ergab folgendes:

1. $0,1132 \mathrm{~g}$ Substanz gaben $31,2 \mathrm{ccm}$ Stickstoff bei $15^{\circ}$ und $759 \mathrm{~mm}$ Bar., d. i. $32,08 \% \mathrm{~N}$.

2. $0,1320 \mathrm{~g}$ Substanz gaben $0,1596 \mathrm{~g} \mathrm{CO}_{2}$ und $0,0707 \mathrm{~g} \mathrm{H}_{8} \mathrm{O}$; d. i. $32,97 \% \mathrm{C}$ und $5,95 \% \mathrm{H}$.

Berechnet für $\mathrm{C}_{6} \mathrm{H}_{13} \mathrm{~N}_{5} \mathrm{O}_{4}$ : C $32,88 \% ; \mathrm{H} 5,93 \% ; \mathrm{N} \mathrm{31,96 \%}$ Gefunden:

C $32,97 \%$ H $5,95 \%$ N $32,08 \%$.

1) cf. A. Kossel, Diese Zeitschrift, Bd. 25, S. 177. 
Die Substanz hatte mithin die prozentische Zusammensetzung des Nitroarginins. Sie ist in kaltem Wasser wenig, in heißem leicht löslich und krystallisiert beim Erkalten aus. Sie löst sich leicht in verdünnter Salzsäure und Salpetersäure, ebenso in verdünnter Ammoniakflüssigkeit. In Eisessig ist sie selbst in der Siedehitze unlöslich. Die wässerige Lösung reagiert neutral.

Nitroarginin aus Rechtsarginin.

$2 \mathrm{~g}$ Argininnitrat wurden mit einer Mischung von $2 \mathrm{ccm}$ konzentrierter Schwefelsäure und $1 \mathrm{ccm}$ rauchender Salpetersäure, welche $10 \% \mathrm{SO}_{3}$ enthielt, unter Zusatz von $1 \mathrm{ccm}$ rauchender Salpetersäure und unter Abkühlung durch eine Eiskochsalzmischung zusammengerieben, wobei keine vollständige Lösung eintrat. Nach 5 Minuten wurde die Flüssigkeit in eiskaltes Wasser gegossen, mit Baryt übersättigt und durch Einleiten eines Kohlensäurestroms neutralisiert. Aus der vom Niederschlag abfiltrierten und eingedampften Flüssigkeit krystallisierte eine schwerlösliche Masse aus, welche durch Waschen vom Baryumnitrat befreit wurde und deren Menge ungefähr $0,7 \mathrm{~g}$ betrug. Aus der Mutterlauge konnte noch eine weitere Substanzmenge dargestellt werden; immerhin betrug die Ausbeute kaum 50\% der theoretischen.

Die Analyse ergab folgende Werte:

1. $0,1180 \mathrm{~g}$ Substanz gaben $33,05 \mathrm{ccm}$ Stickstoff bei $15,25^{\circ}$ und $750,5 \mathrm{~mm}$ Bar., d. i. $32,20 \% \mathrm{~N}$.

2. $0,1549 \mathrm{~g}$ Substanz gaben $0,1882 \mathrm{~g} \mathrm{CO}_{2}$ und $0,0845 \mathrm{~g} \mathrm{H}_{2} \mathrm{O}$, d. i. $33,14 \% \mathrm{G}$ und $6,06 \% \mathrm{H}$.

Berechnet für $\mathrm{C}_{6} \mathrm{H}_{13} \mathrm{~N}_{5} \mathrm{O}_{4}: 32,88 \% \mathrm{C} ; 5,93 \% \mathrm{H} ; 31,96 \% \mathrm{~N}$ Gefunden: $\quad 33,14 \% \mathrm{C} ; 6,06 \% \mathrm{H} ; 32,20 \% \mathrm{~N}$. 